\title{
Editorial
}

\section{GTH 2022: Sustainable Collaboration}

\author{
Jan Beyer-Westendorf ${ }^{1} \quad$ Ute Scholz ${ }^{2}$ \\ 1 Thrombosis Research Unit, Division of Hematology, Department of \\ Medicine I, University Hospital “Carl Gustav Carus” Dresden, \\ Dresden, Germany \\ ${ }^{2}$ Center of Hemostasis, MVZ Labor Dr. Reising-Ackermann und \\ Kollegen, Leipzig, Germany
}

Hamostaseologie 2022;42:7-8.

Dear colleagues and friends of the German Society of Thrombosis and Hemostasis Research (TH),

\section{Welcome to Leipzig!}

It is our great pleasure to host this year's (rather special) annual GTH Congress.

It is special because it is the $66^{\text {th }}$ congress of our society and you all know the song that suggests that life only just begins at the age of 66 .

And, of course, our congress is special due to the ongoing COVID pandemic. To be honest: as we are writing these lines just 8 weeks before we open the congress we still do not know if our congress will really be one of the first major meetings "in presence" after 2 years of pandemic restrictions, cancellations or virtual interactions. We may have to switch back to "virtual" at the very last minute! Please be assured that we have done our very best to organize a smooth and exciting face-to-face conference, while simultaneously monitoring pandemic developments and decisions by authorities. To this end, we have prepared virtual alternatives for several "bad case" scenarios. This process of flexible decision making will continue up until the closing ceremony on March 4. We hope that everything will work out fine - and apologize for any inconvenience or shortcomings that you may experience.

But beyond its $66^{\text {th }}$ birthday and the insecurities around the pandemic, we believe this congress is special because we set out to create a "sustainable" event, mindful of environmental issues and our societies' "carbon footprint." We hope that you will encounter our sustainability efforts throughout the congress and that our society will continue to make this an important aspect of future planning.

Finally, this year's GTH congress is special because of the program content and structure. From day 1 of our planning,

we wanted to get "our partners" involved, aiming to fulfill our congress motto of "sustainable collaboration." Sessions were jointly planned with neighboring societies (Deutsche Gesellschaft für Angiologie, Deutsche Gesellschaft für Hämato-Onkologie, Deutsche Gesellschaft für Transfusionsmedizin und Immunhämatologie, and Deutsche Gesellschaft für Klinische Chemie und Laboratoriumsmedizin) and topics, chairs and presenters were chosen together with these organizations. The program was structured in daily "topic tracks" to facilitate access to concise, condensed updates over a single morning or afternoon session and, wherever possible, industry symposia were aligned to these program tracks. This will particularly benefit colleagues from other medical fields with an interest in thrombosis and hemostasis issues relevant to their specialization. There will also be room for interdisciplinary exchange of different views and expectations around thrombosis and hemostasis.

In considering the many talks in our conference program, and the review articles selected for this issue, we noticed that our field could, in fact, be surrounded by more than one "pandemic":

The plenary talk of Professor Ageno ${ }^{1}$ will focus on the treatment of atypical clots, a question that seems to have become "pandemic" with the advance of modern imaging techniques and safer anticoagulants. Not only do we see more and more of these clots - current evidence indicates that the benefits of treatment most often outweigh the risks.

The state-of-the-art talk (and review article) of Professor Langer $^{2}$ relates to the management of arterial or venous thromboembolism in thrombocytopenic patients. With the increasing survival of cancer patients undergoing long-term therapies the prevalence of thromboses continues to increase, raising concerns in patients with acute or prolonged thrombocytopenia. But with our advancing understanding of

(c) 2022. Thieme. All rights reserved. Georg Thieme Verlag KG,

Rüdigerstraße 14,

70469 Stuttgart, Germany
DOI https://doi.org/ 10.1055/a-1694-4829. ISSN 0720-9355.

Division of Hematology and Hemostaseology, Department of

Medicine I, University Hospital

"Carl Gustav Carus" Dresden,

Technical University,

Fetscherstrasse 74, D-01307

Dresden, Germany (e-mail: jan.

beyer@uniklinikum-dresden.de). 
the pathomechanisms behind immunologic, hematologic or rheumatologic diseases we also have to advise on anticoagulant treatments in thrombocytopenic non-cancer patients. This talk will address this gap.

A complete change of scenery is provided by our guest speaker Dr. Harriet Etheredge ${ }^{3}$ from South Africa. She is not only an expert on ethical issues in medicine but also very interested in the benefits and risks of social media or digital communication platforms for health professionals. Dr Etheredge kindly agreed not only to hold a plenary talk, but also to interact with us directly in an educational session on March 1. Her review article summarizes the "pandemic" risks of fake news, harassments and pseudo-science surrounding us in our daily work and indicates dangers of the "regulatory vacuum" in this field. It also proposes guidance to stay safe in the digital realm.

Dr. Althaus ${ }^{4}$ and co-authors present their views on the diagnosis of platelet function disorders and the challenges for laboratories. We increasingly gain insights into the complexity of platelet function and the immense task associated with facilitating accurate, standardized laboratory testing in this area. At the same time, we face a "pandemic" of test requests or test results in clinical situations, in which testing may not be indicated. Often, we are asked to interpret test results where we lack information on the assay used, on quality standards of the laboratory behind the test report, on the pre-diagnostic handling of the sample or, even worse, on the medication of the patient. All of these factors can be confounders in interpreting tests results. This session, and the associated article, will help to sort through these challenges and emphasizes the importance of "sustained collaboration" between laboratory and clinician.

In his article, Professor Male $^{5}$ has summarized his plenary talk on the status quo of anticoagulation in pediatric patients. Although this scenario is (fortunately) far from being a pandemic, we have seen more and more clinical trials, real-world data and drug approvals over recent years, helping us to better tailor our treatment decisions to our young patients needs.

Professor Middeldorp ${ }^{6}$ and co-authors present data on the "pandemic" question of when and how to treat pregnant women for thrombophilia or thrombosis. Even with the availability of various guidelines, the answers to these questions are often unclear and recommendations contradictory. With increasing awareness amongst patients and care providers, the threshold to test young women for thrombophilia before or during pregnancy seems to decline. The state-ofthe-art presentation associated with this article will cover some of these aspects in more detail.

The review by Professor Renné ${ }^{7}$ provides insight into current developments in the field of anticoagulation. As we all know, with the availability of safer and "easy-to-use" oral anticoagulants the prevalence of long-term anticoagulation has increased worldwide. Moreover, these drugs are now prescribed to patients with heightened risk for bleeding in whom one would not have dared to use vitamin K antagonists. As a consequence, this "pandemic" use of (generally safer) anticoagulants leads to the paradox of frequent reports of bleeding and the quest for rapidly acting antidotes. This "update on safe anticoagulation" therefore summarizes drug developments in the field that specifically target factor XI or XII inhibition with the goal of achieving clot protection in the future without increasing bleeding risks.

Last but not least, Professor Espinola-Klein ${ }^{8}$ summarizes her state-of-the-art presentation on the combination of antiplatelet and anticoagulant drugs. In parallel to the development of various new anticoagulant and antiplatelet drugs, the cardiovascular medicine advances regarding new indications for these treatments. Not only do we continuously see new stents and other cardiovascular devices that require adjustments of our previous treatments but our better understanding of individual risks and benefits also requires a much more tailored approach to our patients. For instance, the concept of combining antiplatelet and anticoagulant drugs to achieve atheroprotection has recently emerged and its benefits seem to extend beyond the antithrombotic effects that have so far defined this intervention.

Taken together, the $66^{\text {th }}$ GTH congress and this congress issue of review articles were designed to provide both update and new insights into the current state and future developments in our field - with a focus on the interfaces with our partners from other medical specialties, researchers and industry. We hope that you will equally enjoy the congress and the review articles selected here.

Finally, we want to thank all authors who have accepted our invitation to provide their review articles despite very tight timelines and a pandemic workload. Similar thanks go out to the expert reviewers and the editorial team at Thieme for their sustained support in difficult times.

\section{Once Again: Welcome to Leipzig!}

\author{
Ute Scholz \\ Jan Beyer-Westendorf \\ Conflict of interest
}

The authors declare that they have no conflict of interest.

\section{References}

1 Ageno W. Treatment of Atypical Clots. Hamostaseologie 2022; 42 : $10-18$

2 Voigtlaender M, Langer F. Management of Vascular Thrombosis in Patients with Thrombocytopenia. Hamostaseologie 2022; 42: 1928

3 Etheredge HR, Fabian J. Communication in Healthcare: Global challenges in the 21st Century. Hamostaseologie 2022; 42: 29-35

4 Wagner M, Uzun, G, Bakchoul T, Althaus K. Diagnosis of Platelet Function Disorders: A Challenge for Laboratories. Hamostaseologie 2022; 42: 36-45

5 Male C. Anticoagulation in Pediatric Patients. Hamostaseologie 2022; 42: 46-53

6 Middeldorp S, Naue C, Köhler C. Thrombophilia, Thrombosis, and Thromboprophylaxis in Pregnancy: For What and in Whom? Hamostaseologie 2022; 42: 54-64

7 Mailer RK, Kuta P, Renné T. An Update on Safe Anticoagulation. Hamostaseologie 2022; 42: 65-72

8 Espinola-Klein C. When and How to Combine Antiplatelet and Anticoagulant Drugs? Hamostaseologie 2022; 42: 73-79 